\title{
Influence of temperature on the elastic properties of composite materials identified with an inverse method
}

\author{
M. Bottiglieri ${ }^{1,2, a}$ and H. Sol ${ }^{2}$ \\ ${ }^{1}$ Erasmushogeschool Brussel, Department of Industrial Sciences and Technology, Nijverheidskaai \\ 170, 1070 Brussels, Belgium \\ ${ }^{2}$ Vrije Universiteit Brussel, Department of Mechanics of Materials of Constructions, Pleinlaan 2, \\ 1050 Brussels, Belgium
}

\begin{abstract}
This paper presents an inverse method for the identification of the orthotropic engineering constants of composite materials. The vibration behaviour of a freely suspended test plate is used as information source. The material parameters in a finite element model of the test plate are tuned to match the measured resonance frequencies. The automated procedure is simple, accurate and fast and can be executed with temperature control.
\end{abstract}

\section{Introduction}

Standard test methods for the determination of elastic engineering constants like, among others, tensile tests, bending tests, torsion tests, require test specimens in which the stress and strain distribution are homogeneous. Standard test methods have proven their validity to the engineering society already a long time. Standard test methods use material specimens with a well defined geometrical shape described in norms. The specimens are subjected to boundary conditions that lead to known, mostly uniform (stress, strain, velocity, other) fields in the tested materials. A common example is a tensile test in which the stresses and strains in all points of the test beam are assumed to be constant. This assumption allows determining the Young's modulus from the measured tensile force, the cross section value, the original length and measured displacement values with a simple formula.

However, when applied to complex materials like composite materials, some limitations of standard test methods become clear. It is often difficult to obtain the required (homogeneous, uniform) deformation field with suitable test specimens. Material heterogeneity, like is the case in nearly all composite materials, can be a reason. Shear coupling in highly anisotropic materials, misalignment and clamping forces can be other reasons making it difficult to obtain homogeneous stress/strain fields. The manufacturing of long fiber or textile reinforced composite material test bars may disrupt the internal structure of the material that has to be investigated. Peel stresses at the free boundaries of beam specimens with small width can have considerable influence on the test results. Moreover, for composite materials with orthotropic material behavior, different beam specimens

\footnotetext{
a e-mail : massimo.bottiglieri@vub.ac.be
} 
must be machined to measure the Young's moduli, Poisson's ratios and shear moduli in different directions.

The proposed inverse method in this paper avoids most of the above mentioned problems for composite materials by taking relatively large plates as test samples. Because the stress and strain field is no longer homogeneous when using test plates, a so-called inverse method is applied. The principle of the applied inverse method in this paper, called Mixed Numerical Experimental Technique, is to compare measured vibration quantities like natural (also called resonance) frequencies from an experiment on a freely suspended rectangular test plate with computed values using a numerical model of the same test plate (Figure 1). More details about the principle of the method can be found in [1], [2] and [3]

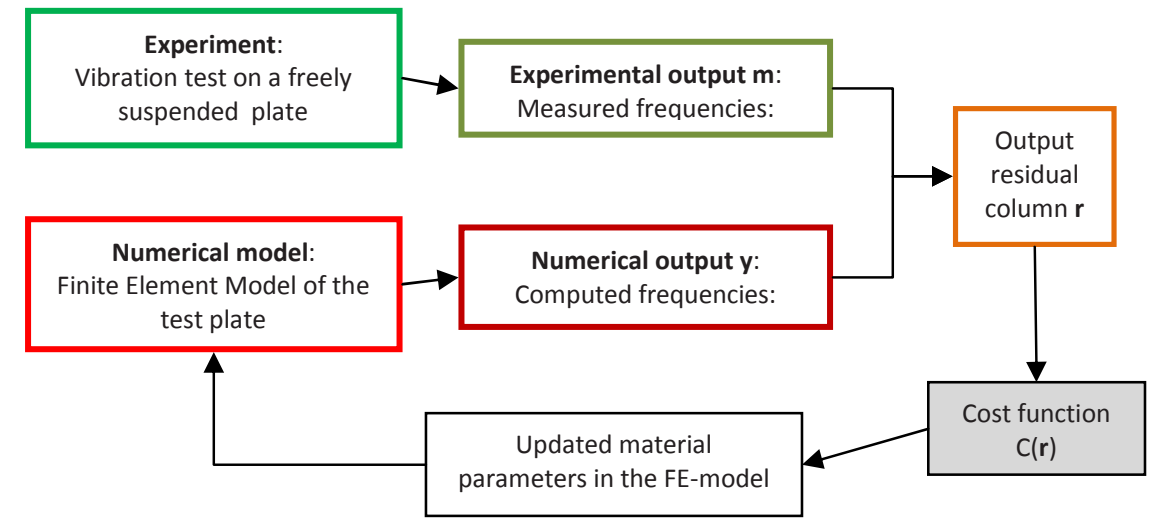

Fig. 1. Principle of an inverse method for material identification (IMMI)

The adopted numerical models are accurate finite element (FE) models of the test plates. The differences between the measured $\mathbf{m}$ ("experimental output") and computed frequency values $\mathbf{y}$ ("numerical output") are stored in a residual column $\mathbf{r}=\mathbf{m}-\mathbf{y}$. The residual column acts as a variable in a scalar cost function $\mathrm{C}(\mathbf{r})$ that describes the goodness of fit between the numerical model output and the measured experimental output. The - a priori unknown - material properties in the inverse method are the parameters in the numerical FE model. Starting from an initial guess, these material properties are iteratively updated until the computed output matches the measured output. The parameter modifications are estimated by minimisation of a scalar cost function. The final result of the output matching procedure is an estimate of the parameters values. The procedure is called "Resonalyser procedure" in the sequel of this paper [4].

\section{The Resonalyser procedure}

\subsection{The principle of the Resonalyser procedure}

The Resonalyser procedure aims at the identification of the in plane orthotropic engineering constants by the measurement of the resonance frequencies of test beams and a rectangular test plate. The procedure is based on the above described principle of inverse methods and results in averaged material stiffness values over the plate and beams areas. The obtained material stiffness values are therefore well suited as input values for e.g. finite element models. The elastic behaviour of orthotropic materials in a plane state of stress can be characterised by 4 independent engineering constants. The engineering constants appear in the symmetric compliance matrix that describes the relation between the strain and stress column. 


$$
\left\{\begin{array}{c}
\varepsilon_{1} \\
\varepsilon_{2} \\
\gamma_{12}
\end{array}\right\}=\left[\begin{array}{ccc}
\frac{1}{E_{1}} & -\frac{v_{21}}{E_{2}} & 0 \\
-\frac{v_{12}}{E_{1}} & \frac{1}{E_{2}} & 0 \\
0 & 0 & \frac{1}{G_{12}}
\end{array}\right]\left\{\begin{array}{l}
\sigma_{1} \\
\sigma_{2} \\
\tau_{12}
\end{array}\right\} \quad \frac{v_{21}}{E_{2}}=\frac{v_{12}}{E_{1}}
$$

In expressions (1), 1 and 2 are the orthotropic material axes; $\varepsilon_{\mathrm{i}}$ is the normal strain component in the $\mathrm{i}$-direction; $\gamma_{12}$ is the shear strain component in the 12-plane, $\sigma_{\mathrm{i}}$ is the normal stress component in the i-direction, $\tau_{12}$ is the shear stress component in the 12-plane, $v_{12}$ and $v_{21}$ : are the major and minor Poisson's ratios in the 12-plane, $E_{i}$ is the Young's modulus in the i-direction and $G_{12}$ is the shear modulus in the 12-plane. Due to the symmetry relation in equation (1), the full characterization of orthotropic material hence requires the evaluation of 4 independent engineering constants.

The Resonalyser procedure proceeds in three steps: the first step establishes a test plate from which the resonance frequencies are sensitive for variations of all the engineering constants. By cutting two test beams along the orthotropic axes (see Figure 2) and measuring the natural frequency $\mathrm{f}_{\mathrm{i}}$ of the freely suspended test beams, it is possible to establish the aspect ratio of a suitable test plate

$$
\text { Aspect ratio Poisson plate }=\frac{L}{W}=\frac{L_{1}}{L_{2}} \sqrt{\frac{f_{1}}{f_{2}}}
$$

In formula (2), $\mathrm{L}$ and $\mathrm{W}$ are the length and width of the test plate. The first natural frequency of each freely suspended test beam is represented by $f_{i} . L_{1}$ and $L_{2}$ respectively represent the length of "Beam 1" and "Beam 2" as shown in Figure 2 below. A plate with an aspect ratio according to formula (2) is called a Poisson plate [4]. The mode shapes associated to the first five natural frequencies of a Poisson plate are shown in Figure 3.

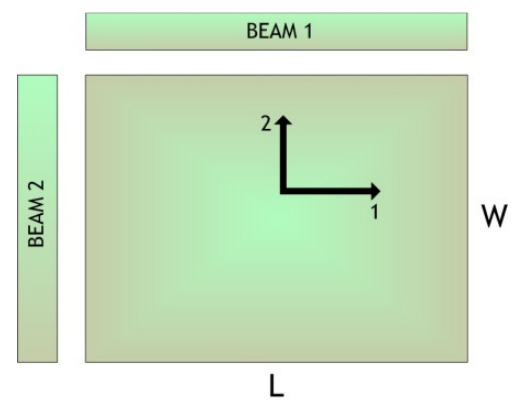

Fig. 2. Cutting two test beams

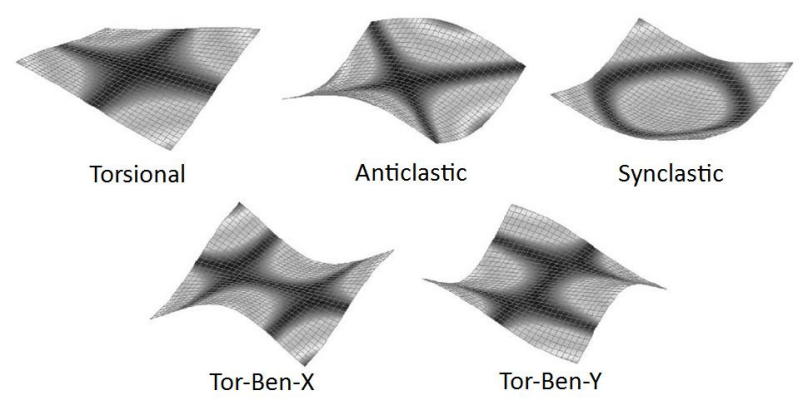

Fig. 3. Mode shapes of a Poisson plate [3]

The frequencies of the anticlastic and synclastic mode shapes of a Poisson plate are especially sensitive for variations of Poisson's ratio [5].

The second step in the Resonalyser procedure consists of the actual measurement of the natural frequencies, while the third step performs the identification of the engineering constants using the inverse method. 


\subsection{Identification of the engineering constants as a function of the temperature}

The 4 unknown material parameters can be stored in a parameter column $\mathbf{p}$. The estimation of $\mathbf{p}$ by updating the finite element model is obtained using the iterative procedure described in Figure 1. The procedure yields good results if the parameters can be sufficiently well "observed" through the measured resonance frequencies. This means that the selected resonance frequencies must be sensitive for variations of each of the 4 desired material parameters.

The first natural frequency of the beam cut along the orthotropic 1 axe is sensitive for variations of $E_{1}$. The first natural frequency of the beam cut along the orthotropic 2 axe is sensitive for variations of $E_{2}$. The first natural frequency of the Poisson plate is associated to a torsion mode and is sensitive for variations of $\mathrm{G}_{12}$ and the third natural frequency is sensitive to variations of Poisson's ratio. These experimentally measured natural frequencies are stored in the experimental output column $\mathbf{m}$, the corresponding frequencies computed with the finite element model of the Poisson plate (and simple beam formulas) using an initial guess for the engineering constants are stored in the computed output column $\mathbf{y}$. Thin beam (Euler-Bernouilli) and thin plate (Love-Kirchhoff) behavior is assumed [6]

$$
\begin{gathered}
\mathbf{p}=\left\{\begin{array}{c}
E_{1} \\
E_{2} \\
G_{12} \\
v_{12}
\end{array}\right\} \quad \mathbf{m}=\left\{\begin{array}{c}
f_{\text {Beam } 1} \\
f_{\text {Beam } 2} \\
f_{\text {Plate Torsion }} \\
f_{\text {Plate Synclastic }}
\end{array}\right\}_{\text {Experimental }} \mathbf{y}=\left\{\begin{array}{c}
f_{\text {Beam } 1} \\
f_{\text {Beam } 2} \\
f_{\text {Plate Torsion }} \\
f_{\text {Plate Synclastic }}
\end{array}\right\}_{\text {Computed }} \\
\mathbf{r}=\mathbf{m}-\mathbf{y}
\end{gathered}
$$

The cost function $C(\mathbf{r})$ is the least squares value of the residue $\mathbf{r}$. $C(\mathbf{r})$ is minimized by updating the values of $\mathbf{p}$.

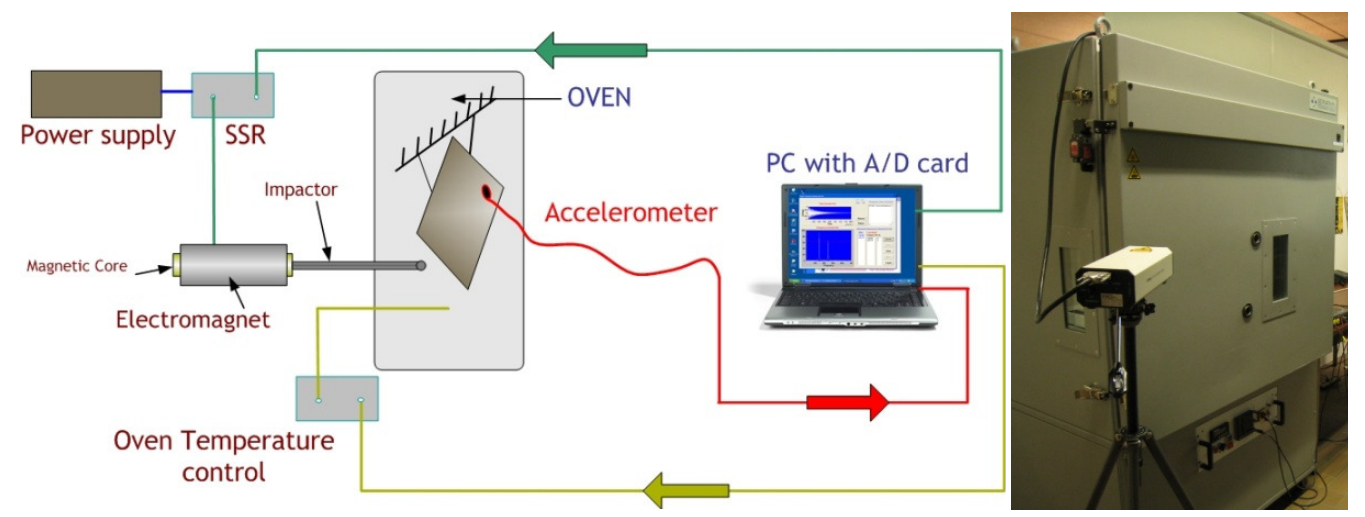

Fig. 4. The measurement test setup.

As can be seen in Figure 4, the test specimens can be placed in a temperature controlled unit. Liquid Nitrogen is used to obtain low temperatures and heated air to obtain high temperatures. The unit's temperature is controlled in a simple way via a remote voltage input generated by PC software. 


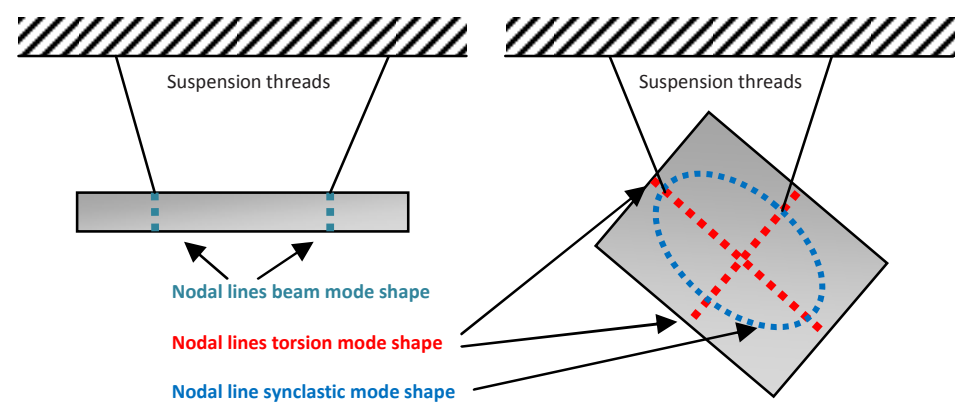

Fig. 5. Suspension the test objects in the nodal lines.

The measurement itself consists of giving an impulse excitation to the freely suspended test specimens and recording the impulse response with an accelerometer. The natural frequencies can be extracted by taking the Fourier transform of the impulse response and standard signal analysis procedures. The excitation is executed by an electromagnet controlled via the PC software.

The test beams and test plates are suspended by quartz threads in the nodal lines of the vibration modes. In this way, the free vibration of the test object has a minimal influence of the suspension threads (see Figure 5).

The measurement starts with the registration of the frequencies for the first bending mode of test beam 1 in a selected temperature range, followed by the registration of the frequencies of test beam 2 in the selected temperature range. The averaged values of both frequency sets are used to establish the aspect ratio of the Poisson test plate with Equation 2. Consequently, the test plate is machined and the first and third natural frequency of the Poisson plate is registered in the selected temperature range. All these measured frequencies are then processed temperature step by temperature step with the inverse method to identify the engineering constants.

Due to the extra mass of the accelerometer, differences might occur on the natural frequencies of the test plate. [7] The experimental natural frequencies will tend to be different from the ones obtained in the numerical model, resulting in an incorrect material parameter identification. Thus, prior to all measurements, a coefficient for the mass compensation has to be determined via an inverse method to eliminate the influence of the mass of the accelerometer. This inverse method will try to obtain matching natural frequencies between the experimentally and numerically obtained results by tuning the mass matrix $[\mathrm{M}]$. Therefore, the position of the sensor (node) and a measurement with an LDV (Laser Doppler Velocity meter) - no additional mass on plate - are required at room temperature. Note that a constant coefficient is assumed for the whole temperature range. 


\section{Example}

The Resonalyser procedure for the identification of the engineering constants is illustrated for Twintex ${ }^{\circledR}[8]$ thermoplastic composite material.

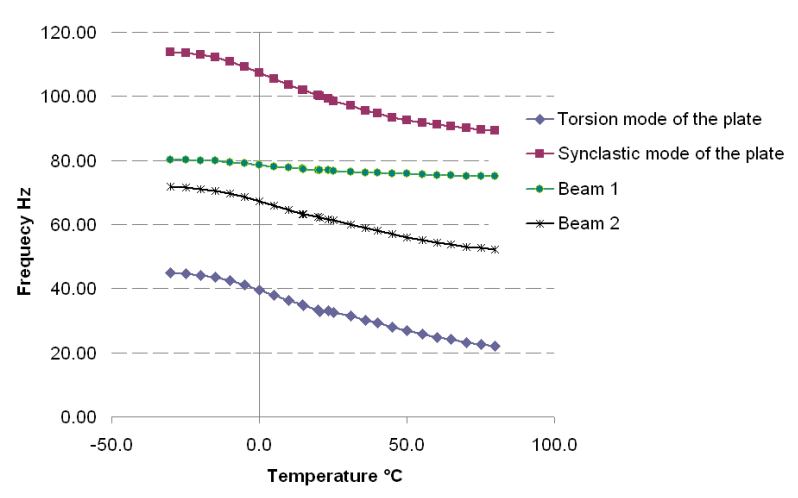

Fig.6. Measured natural frequencies as a function of the temperature.
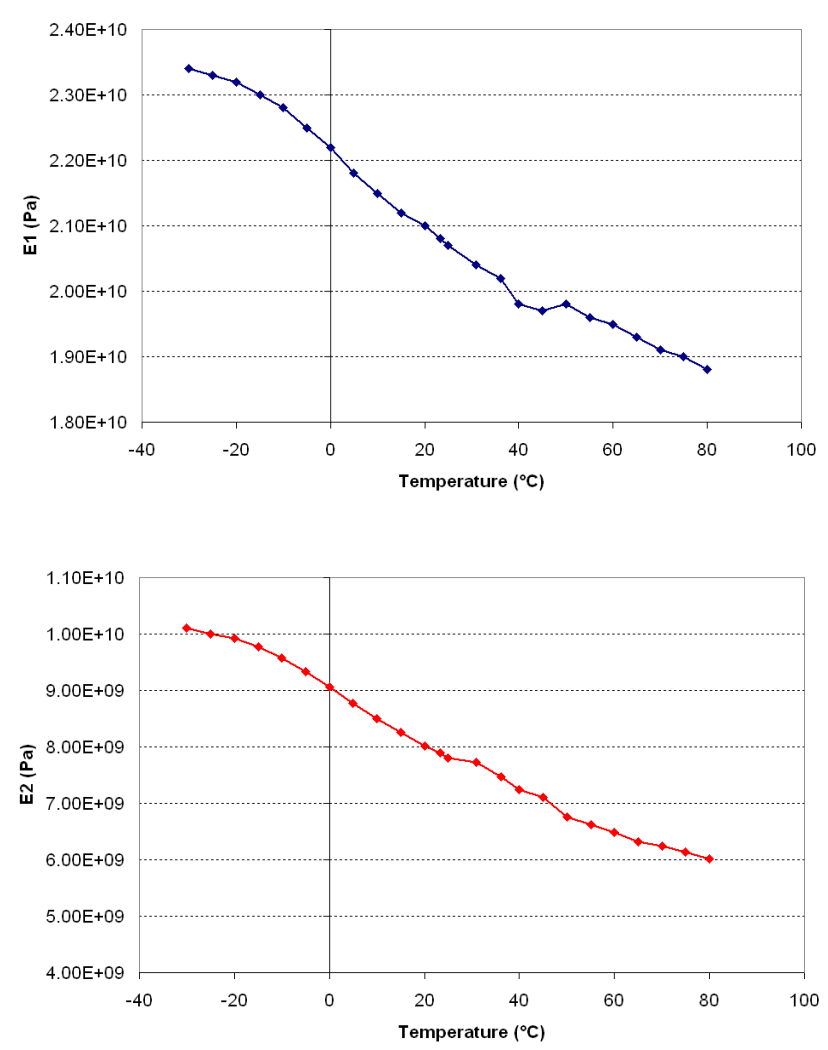

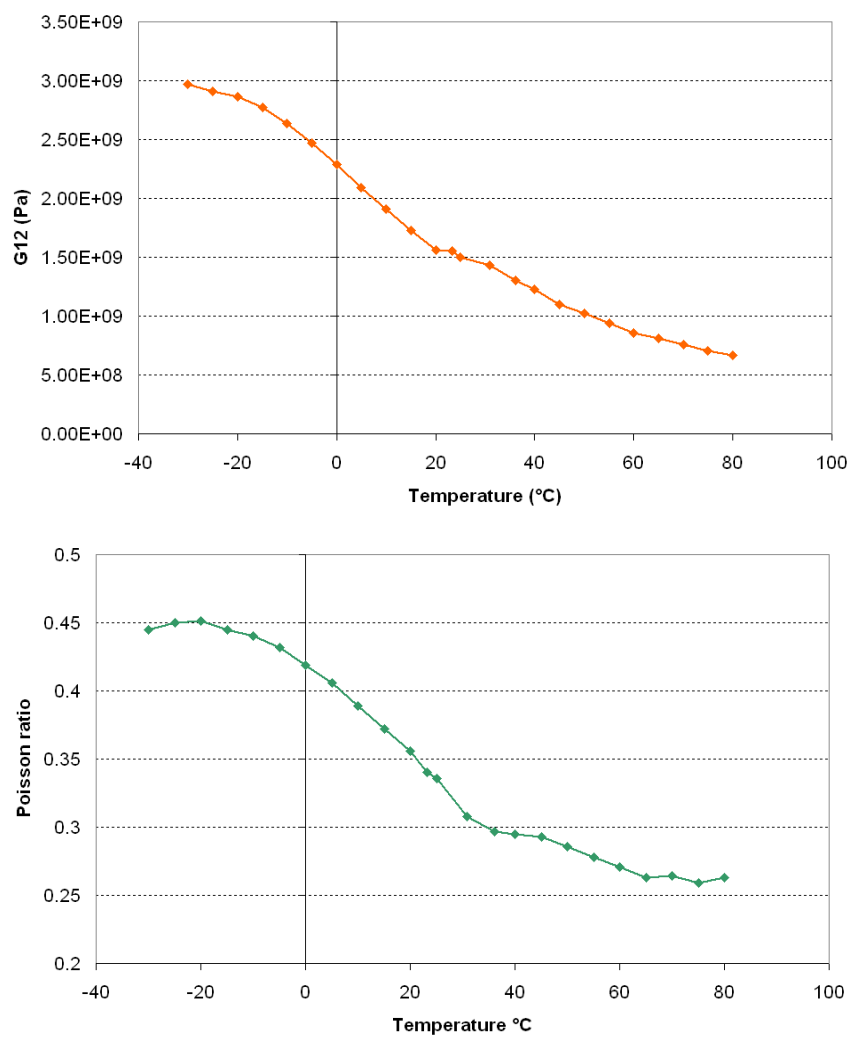

Fig. 7. Identified engineering constants as a function of the temperature.

Twintex ${ }^{\circledR}$ composite material is made with fabrics or roovings composed of commingled E-glass and polypropylene (PP) filaments. Consolidation is done by heating above the melting temperature $\left(180^{\circ} \mathrm{C}-230^{\circ} \mathrm{C}\right)$ of the PP matrix and applying a pressure. Twintex ${ }^{\circledR}$ is used in many automotive, marine and leisure products because of its low mass, good impact resistance and other good mechanical properties. Examples are car bumpers, truck floors, boat frames, snowboards and many other products.

The test plate used for this paper is composed out of 6 layers of bi-directionally woven commingled fabric with $60 \%$ glass fibres. The elastic properties of this thermoplastic composite material are clearly dependent on the temperature. Figure 5 shows the measured frequencies of the two beam specimens and the frequencies of the Poisson plate in the selected temperature range of $30^{\circ} \mathrm{C}$ till $80^{\circ} \mathrm{C}$. The measured frequencies vary monotonously as a function of temperature as can be seen in Figure 6. At temperatures higher than $80^{\circ} \mathrm{C}$, the increased damping value of the test specimens made it impossible to measure the resonance frequencies with the used impulse test procedure.

The identified engineering constants as a function of temperature are plotted in the 4 graphs shown in Figure 7, respectively representing $\mathrm{E}_{1}, \mathrm{E}_{2}, \mathrm{G}_{12}$ and $v_{12}$. The $\mathrm{E}_{1}$ modulus at $-40^{\circ} \mathrm{C}$ is $23 \mathrm{GPa}$ and drops continuously to $19 \mathrm{GPa}$ at $80^{\circ} \mathrm{C}$ (minus $20 \%$ ). The $\mathrm{E}_{2}$ modulus at $-40^{\circ} \mathrm{C}$ is $10 \mathrm{GPa}$ and drops continuously to $6 \mathrm{GPa}$ at $80^{\circ} \mathrm{C}$ (minus $40 \%$ ). The $\mathrm{G}_{12}$ modulus of $3 \mathrm{GPa}$ at $-40^{\circ} \mathrm{C}$ is clearly matrix dominated and drops with a factor 5 to a low fibre dominated value of $0.6 \mathrm{GPa}$ at $80^{\circ} \mathrm{C}$. The Poisson ratio at $-40^{\circ} \mathrm{C}$ is matrix dominated and has a value of 0.45 . At $80^{\circ} \mathrm{C}$ the Poisson ration has the typical low value of 0.25 of bi-directionally reinforced composite materials 


\section{Conclusion and error discussion}

The 4 measured resonance frequencies of the test beams and test plate contain enough information to identify the desired engineering constants by an inverse method using an accurate finite element plate model. Error discussion about material identification using inverse methods is a difficult matter because the final results are influenced by model errors as well as by measurement errors. If it is assumed that the material properties are uniform over the test plate area and if the finite element model of the plate is considered as very precise, then the accuracy is mainly determined by the measurement error. In the Resonalyser procedure the estimation is based on the measurement of resonance frequencies. The experimental error on measured frequencies is small, even if nonsophisticated measurement equipment is used. Reference testing and comparing Resonalyser results with results from standard testing have proven that the identified engineering constants are very accurate [9]

It is not yet investigated if the possible non-uniform temperature distribution in the test plate must also be considered as a source of error.

\section{References}

$1 \mathrm{H}$. Sol, Identification of anisotropic plate rigidities using free vibration data, $\mathrm{PhD}$ Thesis, Vrije Universiteit Brussel, Belgium, (1986).

2 H. Sol, H. Hua, J. De Visscher, J. Vantomme, W.P. De Wilde, A mixed numerical/experimental technique for the nondestructive identification of the stiffness properties of fibre reinforced composite materials, Journal of NDT\&E International, 30, Elsevier Science Ltd, pp. 85-91, (1997).

3 Lauwagie, T., Sol, H., Roebben, G., Heylen, W., Shi, Y., Van der Biest, O., Mixed numericalexperimental identification of elastic properties of orthotropic metal plates, Journal of NDT\&E International, 36, pp. 487-495, (2003)

4 Sol, H., De Visscher, J., Hua, H., Vantomme, J., De Wilde, W.P., La procedure Resonalyser, La Revue des laboratoires d'essais, 46, pp. 10-12, (1996)

5 T. Lauwagie, K. Lambrinou, H. Sol, W. Heylen, Resonant-Based Identification of the Poisson's Ratio of Orthotropic Materials, Journal of Experimental Mechanics, DOI 10.1007/s11340-0099250-9, (2009)

6 Timoshenko, S., Theory of Plates and Shells, McGraw-Hill Book Company, 1st Ed., (1940).

7 D.J. Ewins, Modal Testing: theory, practice and application, Research Studies Press Ltd., (1989).

8 Twintex ${ }^{\circledR}$, www.owenscorning.com, (2009)

9 Lauwagie, T., Sol, H., Roebben, G., Heylen, Handling uncertainties in mixed numericalexperimental techniques for vibration based material identification", Journal of Sound and Vibration, Volume 291, Issues 3-5, Pages 723-739, (2006) 